\title{
The rule governing the abundances of cosmic rays at the sources prior to acceleration
}

\author{
Antonio Codino* \\ INFN Perugia \\ E-mail: antonio.codino@pg.infn.it
}

Cosmic ray abundances, $\mathrm{A}_{c r}$, are compared with those of quiescent matter in the Galaxy, $\mathrm{A}_{g}$, for 40 nuclei from Hydrogen to Zirconium via the abundance ratios $\mathrm{A}_{c r} / \mathrm{A}_{g}$. Secondary nuclei generated by spallation reactions in the interstellar medium are removed from the analysis reducing the sample to primary nuclei. The abundance ratios of primary nuclei obey a simple rule depending on the atomic number $\mathrm{Z}$, namely $\mathrm{A}_{c r} / \mathrm{A}_{g}=\mathrm{aZ}-\mathrm{b} \mathrm{Z}$ where $\mathrm{a}$ and $\mathrm{b}$ are numerical constants. This study suggests that the physical mechanism giving the correct, quantitative explanation of the above rule is the photoionization of the quiescent, cold interstellar matter. The ultraviolet light spectrum causing photoionization of neutral atoms is highly characteristic and concentrated in a very restricted band, namely $3-250 \mathrm{~nm}$. It is argued that such an ultraviolet spectrum and the related absolute intensity can be generated by $\mathrm{O}$ and $\mathrm{B}$ stars. It is concluded that the sources of cosmic rays are located in cold zones, at adequate distances from $\mathrm{O}$ and $\mathrm{B}$ stars beyond the fully ionized Strömgren shells. Previous explanations of the universal $\mathrm{A}_{c r} / \mathrm{A}_{g}$ abundance ratios are discussed.

36th International Cosmic Ray Conference -ICRC2019-

July 24th - August 1st, 2019

Madison, WI, U.S.A.

* Speaker. 


\section{Introduction}

Cosmic ray energies are measured up to $2 \times 10^{20} \mathrm{eV}$ [1]. The discovery of the heavy chemical composition in the utmost energy range $3 \times 10^{18}-10^{20} \mathrm{eV}[2,3]$ has further demonstrated the galactic origin of cosmic rays according to an exploratory interpretation $[4,5,6]$ of the chemical composition data $[2,3,7]$. Nevertheless, a widespread, persistent and disputable tradition still admits an extragalactic origin above $6 \times 10^{19} \mathrm{eV}$.

The galactic origin of cosmic rays prompts the quest for the galactic regions which emanate cosmic rays before acceleration. More precisely, the quest for the minute fractions of the huge galactic volume of $1065-1066 \mathrm{~cm}^{3}$ characterized by specific material composition and physical mechanisms selecting the observed, distinctive abundances of fully ionized nuclear species of the cosmic radiation. These minute fractions of the galactic volume filled with a specific blend of neutral and ionized atoms have been shortly termed sources.

Thus, the source notion incorporates both a geographical concept and a sieve mechanism. Efforts to identify cosmic-ray sources have been reported in the literature since decades but a unanimous, irrefutable consensus has yet to come. Presently the best solution to the cosmic-ray source problem comes from the arena of isotopic and elemental abundances of cosmic rays [8,9]. Two independent lines of arguments indicates that cosmic-ray sources coincide with the galactic regions where $\mathrm{O}$ and $\mathrm{B}$ star associations inhabit. The arguments hinge on the isotopic abundance of ${ }^{22} \mathrm{Ne}$ $/{ }^{20} \mathrm{Ne}$ species [8], which overwhelms by a factor $5.3 \pm 0.3$ that of the undifferentiated interstellar medium, and on the comparison of quiescent galactic matter with cosmic-ray nuclei [9].

This communication describes an attempt to localize cosmic-ray sources and identify the sieve mechanism. For clarity the result is anticipated here : cosmic-ray matter prior to acceleration resides in cold, thick galactic regions surrounding $\mathrm{O}$ and $\mathrm{B}$ star associations and it is selected by photoionization of ultraviolet light of star and extrastar origin. Ultraviolet light transforms a fraction of neutral atoms at low temperature in the proximity of $\mathrm{O}$ and $\mathrm{B}$ stars in ionized atoms, which coexist with neutral atoms. From this blend of ionized material originates galactic cosmic rays. A silent assumption is that the acceleration mechanism, regarded as unknown in this work, does not alter the abundances in the cosmic-ray sources.

Galactic abundances are denoted by $\mathrm{A}_{g}$ while cosmic-ray abundances by $\mathrm{A}_{c r}$ and, accordingly, the abundance ratio or quotient by $\mathrm{A}_{c r} / \mathrm{A}_{g}$ and, concisely, $\mathrm{r} \equiv \mathrm{A}_{c r} / \mathrm{A}_{g}$ which is a function of the atomic number, $\mathrm{Z}$. The observation of a common spectral index of $2.67 \pm 0.05$ for eleven cosmic nuclei in a large energy band $10^{11}-10^{15} \mathrm{eV}$, reported in 2012 by the TRACER experiment [10] is a notable empirical achievement in Cosmic Ray Physics and is adopted in this work as ordo rerum. The cosmic Iron is taken as normalization flux at the arbitrary energy of $2.24 \times 10^{15} \mathrm{eV}$ just below the knee position. At this energy the flux is taken to be $3.14 \times 10^{-13}$ particles $/ \mathrm{m} \mathrm{s}$ sr $\mathrm{GeV}$ consistent with numerous data. Neither the specified flux nor the energy of $2.24 \times 10^{15} \mathrm{eV}$ are critical parameters in the interval of $10^{12}-3 \times 10^{15} \mathrm{eV}$ and are specified for clarity.

Cosmic-ray abundances of $\mathrm{H}, \mathrm{He}, \mathrm{C}, \mathrm{N}, \mathrm{O}, \mathrm{Ne}, \mathrm{Mg}, \mathrm{Si}, \mathrm{S}, \mathrm{Ca}$ and $\mathrm{Fe}$ are based on recent measurements [11,12] while those of more rare elements are taken from previous measurements [13] and compilations [14]. The ratio $r$ is expressed in total energy per nucleus instead of energy 
per nucleon or energy per atomic mass unit as routinely done in all past literature. Abundances are evaluated at the energy of $10^{13} \mathrm{eV}$ and not below, due to the high quality of the recent data $[11,12]$. The unit of total energy per nucleus and the evaluation energy of $10^{12} \mathrm{eV}$ have three notable advantages : (A) the correction of the energy spectrum for the solar modulation becomes thoroughly negligible; (B) the conversion of the abundances measured in the detector into those of the sources are simpler. For instance, cross sections for cosmic-ray propagation in the Galaxy at low energies, in the range $0.5-2 \mathrm{GeV}$, are a jungle of bumps, which in some cases are not well measured or remain unmeasured. (C) In the range $10^{12}-10^{15} \mathrm{eV}$ cosmic-ray spectrum has a constant index $\gamma$ of $2.67 \pm 0.05[10]$ while in the lower and higher adjacent energy regions the spectral index varies. Accordingly, to solve the source problem, the obvious starting point, is to evaluate abundances at the energy of $10^{13} \mathrm{eV}$ to get rid of circumstantial subproblems (A) and (B) and benefit from a constant spectral index as noted in $(\mathrm{C})$.

\section{The rule governing the ratio of the galactic to solar abundances}

The elemental abundances of the Sun are regarded as representative of those of the undifferentiated interstellar matter according to a multidecennial tradition in the investigation of $\mathrm{A}_{c r} / \mathrm{A}_{g}$ ratio. This paper follows this tradition, yet some caveats and elucidations are shortly discussed. Two notorious compilations report the atmospheric abundances of the Sun [15] and meteoritic abundances of the protosolar system [16]. Presently the Sun occupies a low density cavity [for example see ref. 17] with zigzag boundaries sized about 100-150 pc believed to be excavated by active stars, novae and supernovae explosions, and internal dynamics of clouds in past epochs. The interstellar medium around the Sun within a reach of about $100 \mathrm{pc}$ has elemental abundances similar to the B star ambient which is underendowed in heavy elements [18], by a factor 1.5-2, with respect to solar abundances. Notice that Sun abundances are also elevated with respect to those of G and F main sequence stars in the disc [19]. Empirical evidence testifies that some atoms encapsulated in grains in the interstellar medium are scarce both in the atmospheres of the main sequence stars and in the gas phase of the undifferentiated interstellar medium. These atoms are, primarily, Carbon and Oxygen, and secondarily, Silicon, Magnesium and Iron. A quantitative evaluation of atom abundances in grains depends on the grain model whose main five parameters are mass density, size distribution, static dielectric constant, porosity and age. Presently abundance estimates in grains are model dependent and disparate grain compositions have been calculated [for instance see ref. 18].

The observed enrichment of heavy elements in the galactic disc in the last 4-5 Gigayears, monitored by the $[\mathrm{Fe}] /[\mathrm{H}]$ observations and others, should not play an important role in the evaluation of $\mathrm{A}_{c r} / \mathrm{A}_{g}$ quotients since the age of cosmic rays is in the range 10-20 million years. The conclusion from this brief scrutiny is that the photospheric abundances of the Sun are a legitimate surrogate of the undifferentiated interstellar medium of the Galaxy within a factor 2. Notice that the factor 2 is a one-way indicator : heavy elements starting from Carbon are more abundant in the Sun than in $\mathrm{B}$ star ambient. The $\mathrm{A}_{c r} / \mathrm{A}_{g}$ ratio versus atomic number $\mathrm{Z}$ is shown in figure 1 in the range $1 \leq \mathrm{Z} \leq$ 28.

The fundamental properties of the $\mathrm{A}_{c r} / \mathrm{A}_{g}$ ratios are discussed in a previous paper [20]. Nuclei with odd atomic numbers ( $Z=5,7,9,11,13,15,17$ and 19) have to be disregarded in this 
study because they are secondary nuclei of the cosmic radiation copiously produced by spallation reactions in the interstellar medium. These nuclei leave an indelible mark on the function $\mathrm{A}_{c r} / \mathrm{A}_{g}$ consisting in a systematic superabundance of odd nuclei relative to the adjacent even nuclei ( $Z=6$, $8,10,12,14,16,18$ and 20$)$. The effect is redundantly proved by the opposite, distinctive zigzag in the function $\mathrm{A}_{g}$ in the same range $5 \leq \mathrm{Z} \leq 20$ where odd nuclei are underabundant relative to adjacent overabundant even nuclei (compare fig. 1 and 3 of ref. 20).

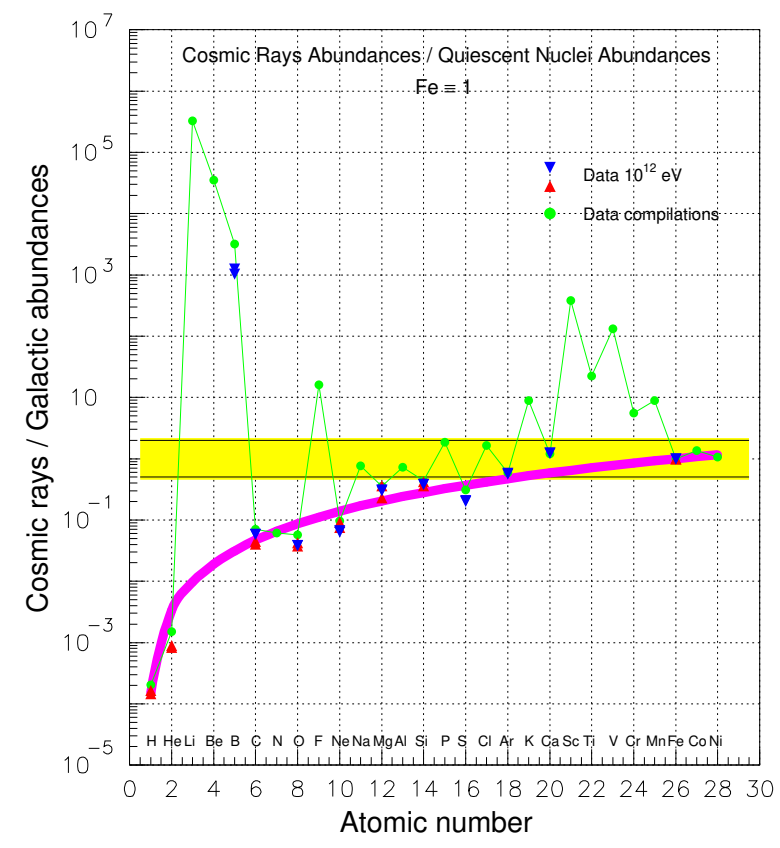

Figure 1: The $\mathrm{A}_{c r} / \mathrm{A}_{g}$ ratios versus atomic number $\mathrm{Z}$ (even) from Hydrogen to Nickel. Only primary species ( located on the thick pink curve) have to be taken into account in the search for cosmic-ray sources. Dominant secondaries are the very rare galactic elements $\mathrm{Li}, \mathrm{Be}$ and $\mathrm{B}$, spallation elements close to the $\mathrm{Fe}$ group, namely Sc, Ti, Va, Cr and Mn, along with odd nuclei B, F, Na, Al, P, Cl and K. Numerical values in the figure come from the galactic abundances $[15,16]$ and cosmic-ray data $[11,12,13]$ and compilation [14].

\section{Cosmic ray to galactic abundance ratio versus atomic number}

The $\mathrm{A}_{c r} / \mathrm{A}_{g}$ ratios from Hydrogen to Nickel of fig. 1 (pink curve) are interpolated by the equation

$$
r \equiv A_{c r} / A_{g}=a_{1} Z^{2}-a_{2} Z
$$

where $a_{1}=1.53 \times 10^{-3}$ and $a_{2}=1.4 \times 10^{-3}$. Nuclei of the quiescent matter at the cosmic-ray sources, before being accelerated, are sieved : higher is the atomic number $\mathrm{Z}$ lower is the sieve. Hence, in the range $1 \leq \mathrm{Z} \leq 28$ proton is the most sieved nucleus while Nickel atoms are almost unaffected by the filter. This fact is summarized by a parabola in $Z$, namely the equation (3.1).

The search for the physical mechanism inherent the rule of the equation (3.1) (thick pink curve in fig. 1) takes the cue from the unobserved anticorrelation between first ionization potentials $I(Z)$ 
and $\mathrm{A}_{c r} / \mathrm{A}_{g}$ abundance ratios of $\mathrm{H}$ and $\mathrm{He}$ nuclei. In fact the first ionization potential of Hydrogen is, $\mathrm{I}(1)=13.6 \mathrm{eV}$, and that of Helium, $\mathrm{I}(2)=24.6 \mathrm{eV}$; the abundance ratios are, respectively, $\mathrm{A}_{c r}(1) /$ $\mathrm{A}_{g}(1)=1.3 \times 10^{-4}$ and $\mathrm{A}_{c r}(2) / \mathrm{A}_{g}(2)=1.0 \times 10^{-3}$. The correlation consists in the hierarchy, $\mathrm{I}(1)<$ $\mathrm{I}(2)$ and $\mathrm{A}_{c r}(1) / \mathrm{A}_{g}(1)<\mathrm{A}_{c r}(2) / \mathrm{A}_{g}(2)$. The opposite trend, that is an anticorrelation, is obeyed by any pair of heavier nuclei, for instance Oxygen and Iron. The first ionization potential of oxygen is $\mathrm{I}(8)=13.61 \mathrm{eV}$ and the abundance ratio $\mathrm{A}_{c r}(8) / \mathrm{A}_{g}(8)=0.04$; the first ionization potential of Iron is $\mathrm{I}(26)=7.90 \mathrm{eV}$ and the abundance quotient is $\mathrm{A}_{c r}(26) / \mathrm{A}_{g}(26) \equiv 1.0$. Hence, the anticorrelation, $\mathrm{I}(8)>\mathrm{I}(26)$ and $\mathrm{A}_{c r}(8) / \mathrm{A}_{g}(8)<\mathrm{A}_{c r}(26) / \mathrm{A}_{g}(26)$ is opposite to the $\mathrm{H}$ and He correlation. Since galactic matter and cosmic rays are dominated by $\mathrm{H}$ and $\mathrm{He}$, these numerical figures prove that $\mathrm{r}$ versus $\mathrm{I}(\mathrm{Z})$ are not correlated nor anticorrelated.

Although the $\mathrm{A}_{c r} / \mathrm{A}_{g}$ ratios versus first ionization potentials do not follow a tight and universal anticorrelation, a potent but elusive connection of the two quantities is undeniable in the range 1 $\leq \mathrm{Z} \leq 28$, as reported in the literature for more than fifty years. Since relative abundances of ions produced by photoionization has a definite relation with the first ionization potentials, it is worth exploring in detail this avenue and to determine to what extent photoionization may account for the abundance data in figure 1 (thick pink curve).

\section{Calculations of photoionization cross sections}

Photons collide with electrons bound in atoms of the interstellar matter and ionize them. The aim of this calculation is to determine the chemical composition of the ionized matter resulting from photoionization of neutral cold interstellar matter. The calculation is performed in the thick approximation target specified below. Let be $\sigma(\mathrm{Z}, v)$ or simply $\sigma$ the photoionization cross section of the $\mathrm{Z}$ element at the photon frequency $v$ and $\phi(v)$, photon intensity. The values of $\sigma(\mathrm{Z}, v)$ are in the range of $10^{-18} \mathrm{~cm}^{2}$ with ultra-steep dependence on $v$ at threshold energies due to a quantum mechanical effect. Photoionization cross sections of Hydrogen, Helium, Oxygen and Ca III are given in Fig. 2. Adequate interpolations of the cross sections are utilized with parameters taken from known compilations [21,22].

There are 35 categories of stars but only those with temperatures 40000-100000 degrees yield the copious ultraviolet light capable of ionizing cold neutral matter, carving the $\mathrm{H}$ II regions with typical size of 1-50 parsec (Strömgren radii).

Ideal black body spectra emitted from photospheres of $\mathrm{O}$ and $\mathrm{B}$ stars are disfigured by the absorption lines in the stellar atmospheres and in the cold mantle of dust and gas surrounding the star, approximately in the 0.5-3 parsec range.

The photon spectrum from the star is designated by $\phi_{1}(v)$ in the range $1 \times 10^{15} \leq v \leq 10 \times$ $10^{15}$ cycles/s .

Unequivocal empirical evidence shows that stellar winds emit an ultraviolet spectrum debouching in the soft $\mathrm{X}$ ray band, denoted here by $\phi_{2}(v)$ in the frequency domain $v \geq 10^{16} \mathrm{cy}-$ cles/sec. This spectrum causes the ejection of the deepest shell electrons contributing to the chemical composition of the ionized matter as well. Hence, this photon spectrum has to be added above $v \geq 10^{16}$ cycles/s to the stellar spectrum $\phi_{1}(v)$. The resulting photon spectrum is, $\phi(v)=\phi_{1}(v)+$ $\phi_{2}(v)$ where $\phi(v)$ is the global ionizing photon spectrum impinging on the cold interstellar matter located a few parsec from the hot star. The input spectrum assumes a Rayleigh-Jeans tail of a black 
body profile [23] with an extension to $120 \times 10^{15}$ cycles/s $(495 \mathrm{eV})$ to include ultraviolet light of extrastar origin. Photons of the $\phi_{1}(v)$ spectrum, after being geometrically diluted as $1 / \mathrm{r}^{2}(\mathrm{r}$ is the distance from the emitting star) will impact on the cold matter surrounding the star and extinguished. More precisely, cold matter thickness in H II regions exceeds the mean collision length of the ionizing photons (thick approximation target).

The results of the calculation are shown in fig. 3. Preliminary calculations indicate that the abundances in the interval range $30 \leq \mathrm{Z} \leq 41$ measured by superTIGER experiment fall in the blue striated rectangle.

If cosmic rays were originated in the Strömgrem regions around the $\mathrm{O}$ and $\mathrm{B}$ stars, where atoms are thoroughly ionized, the chemical composition of the sources would have been the same as that of the quiescent interstellar matter ; in this fictitious, unreal condition the $\mathrm{r}$ ratios would have been equal to unity for any Z. On the contrary, disparate values of $r$, scattered over four order of magnitudes between 1 and $10^{-4}$ (fig. 3) are observed, and they are an imprint of the severe selection mechanism acting upon the cold neutral matter.

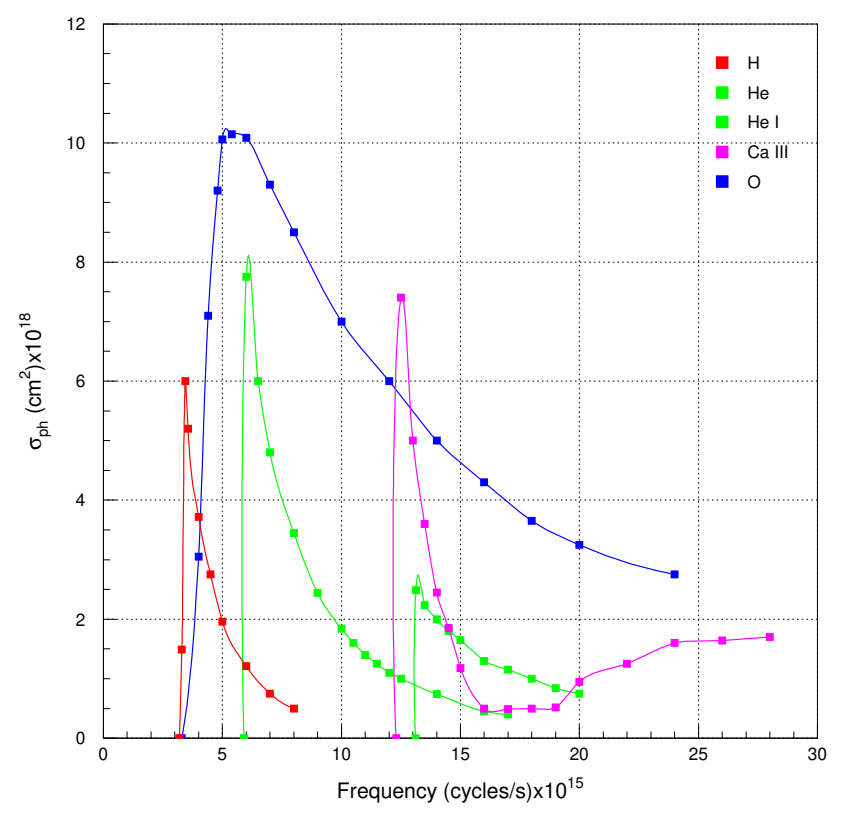

Figure 2: Examples of photoionization cross sections for $\mathrm{H}, \mathrm{He}, \mathrm{O}$ and $\mathrm{Ca}$ III used in the present calculations. The shape of Ca III cross section is the typical interpolation curve given in ref. 21 valid for many other elements

\section{Previous attempts to identify cosmic ray sources}

Although the acceleration mechanism is not absolutely necessary to solve the cosmic-ray source problem, its true nature would have been precious for consistency checks. 


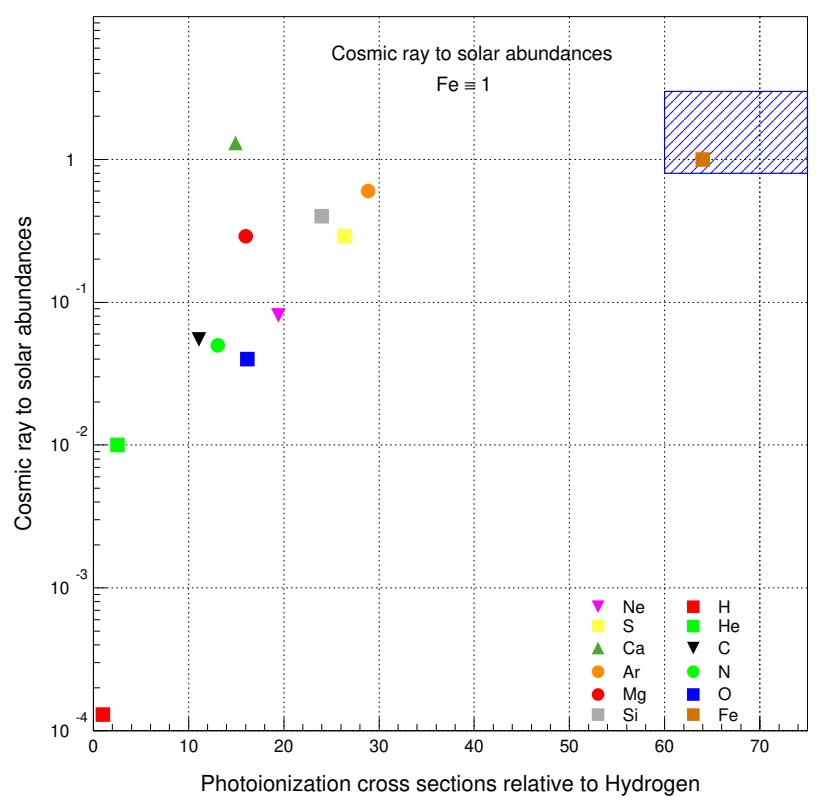

Figure 3: The $\mathrm{A}_{c r} / \mathrm{A}_{g}$ ratios versus photoionization cross sections for some even primary nuclei of the cosmic radiation in the range $1 \leq \mathrm{Z} \leq 26$. Abundances of the elements $30 \leq \mathrm{Z} \leq 40$ lie in the region close to the striated blue rectangle according to preliminary calculations

An interesting example of electromagnetic acceleration appeared in 1972 [24]; magnetic stars were believed to be the sites of cosmic-ray acceleration and the sources were located [25] at adequate distance from the magnetic stars. Circumstellar matter composition resulted from the ionization of energetic electrons (with energies peaked at about $80 \mathrm{MeV}$, an ad hoc hypothesis) and the ensuing $\mathrm{A}_{c r} / \mathrm{A}_{g}$ ratios exhibited an anticorrelation with first ionization potentials. This anticorrelation was believed to agree with the $\mathrm{A}_{c r} / \mathrm{A}_{g}$ ratios expressed in MeV/amu measured at that time (see fig. 2 ref. 25). This model received severe criticism and posed in a limbo. Motivated skepticism came from the unmatchable energy outputs : that of cosmic-ray flux and that delivered by magnetic stars via the conceived mechanism which turned out to be insufficient. This particular model clearly testifies what is desirable to know in terms not only of source location and matter composition, but also on the acceleration process.

The dominant acceleration mechanism recurrent in the literature in the last decades is the diffusive acceleration model believed to take place in supernovae remnants. Cosmic rays would be accelerated by multiple passages, lasting thousand years, through a cobweb of acoustic waves with extremely low energy gains in each passage. In this model cosmic ray sources necessarily coincide with the supernova remnants and the cosmic-ray abundances would be those of the interstellar matter in the same ambient. There is no viable explanation in this model for the great abundance of heavy elements and the paucity of $\mathrm{H}$ and He shown in figure 1. 
In addition, the absence of ${ }^{59} \mathrm{Ni}$ in cosmic ray flux has frontally questioned the hypothetical diffusive acceleration model. This nuclide decays in ${ }^{59} \mathrm{Co}$ with an half life of 74000 years. The number of detected ${ }^{59} \mathrm{Ni}$ in the superTIGER experiment is zero confirming the null result of a previous measurement [26]. As a consequence, the time elapsed between the release of fresh ${ }^{59} \mathrm{Ni}$ from the exploding star and its acceleration has to be greater than 74000 years. Immediately after the star explosion shock waves develop in the remnants and low energy cosmic rays are believed to be accelerated in few thousand years. Hence, an accelerating ${ }^{59} \mathrm{Ni}$ would have no time to decay in ${ }^{59} \mathrm{Co}$, being electron capture extremely unlike for any relativistic nuclide, and so the ${ }^{59} \mathrm{Ni}$ species is expected to intercept terrestrial detectors with a finite flux. But the data [26] are inconsistent with this picture of the diffusive acceleration model.

The two historical examples of acceleration mechanisms mentioned above had a misleading role in the identification of cosmic-ray sources. For instance, the misleading role is evident in the interpretation of ${ }^{59} \mathrm{Ni}$ isotopic abundance data [26], interpretation frequently recurring in the literature. The ad hoc hypothesis conceived in this case to rescue the diffusive acceleration model has been to change the small volumes of supernova remnants into the huge volumes of superbubbles. With this artifice, due to the large size of a typical superbubble, the ${ }^{59} \mathrm{Ni}$ has plenty of time to decay and its absence in the cosmic ray flux would be surreptitiously justified. Superbubbles are galactic regions $10-50 \mathrm{pc}$ in size, excavated by winds and explosive events of very massive stars, where hot and low density matter resides, generally surrounded by a colder and denser interstellar medium.

If the photoionization of cold matter in $\mathrm{O}$ and $\mathrm{B}$ star associations is the dominant mechanism explaining the universal abundance ratios shown in fig. 1, the cosmic-ray sources have to reside at the periphery of these star aggregates. In this case the physical process accelerating cosmic rays is not acoustic, but of electromagnetic nature.

\section{References}

[1] V. Verzi et al. , 2017, Measurement of Energy Spectrum of Ultra-High Energy Cosmic Rays, Progress in Theoretical and Experimental Physics 12 [astro-ph/1705.09111v1] .

[2] M. Unger et al., 2007, Study of the Cosmic Ray Composition above 0.4 EeV using the Longitudinal Profiles of Showers observed at the Pierre Auger Observatory, in proceedings of 30 th ICRC, Merida, Mexico.

[3] R. U. Abbasi et al., 2014, Study of Ultra-High Energy Cosmic Ray Composition Using Telescope Array's Middle Drum Detector and Surface Array in Hybrid Mode [astro-ph/1408.1726v1].

[4] A. Codino, 2017, About the Energy Interval beyond the Ankle Where the Cosmic Radiation Consists Only of Ultraheavy Nuclei from Zinc to the Actinides, Journal of Applied Mathematics and Physics, Vol. 5, pages 225-237.

[5] A. Codino, 2017, The energy spectrum of ultraheavy nuclei above $10^{20} \mathrm{eV}$, Journal of Applied Mathematics and Physics, Vol. 5, pages 1540-1550 [astro-ph/1707.02487v1] .

[6] A. Codino, 2017, About the consistency of the energy scales of past and present instruments detecting cosmic rays above the ankle energy,[arXiv: 1710.06659v1, astro-ph. HE ] .

[7] M. Mallamaci et al. , 2017, Measurements of the depth of maximum muon production and of its fluctuations in extensive air showers above $1.5 \times 10^{19} \mathrm{eV}$ at the Pierre Auger Observatory, in proceedings of $35^{\text {th }}$ ICRC, work 509, Basan, Korea POS (ICRC2017) 509. 
[8] W. R. Binns et al. , 2005, Cosmic-Ray Neon, Wolf-Rayet Stars, and the Superbubble Origin of Galactic Cosmic Rays,ApJ, 634, 351-364.

[9] R. P. Murphy et al ., 2016, Galactic Cosmic Ray Origins and O B Associations : Evidence for SuperTIGER Observations of Elements of ${ }_{26} \mathrm{Fe}$ through ${ }_{40} \mathrm{Zr}, \mathrm{ApJ}, \mathbf{8 3 1}, 148$ (7 pages).

[10] J. P. Boyle et al., 2011, New measurements of the composition and energy spectra of cosmic-ray nuclei with TRACER, in proceedings of 32 ICRC 2011, beijing, China.

[11] M. Ave et al. , 2009, Propagation and source energy spectra of cosmic ray nuclei at high energy, ApJ, 697, 106-114.

[12] H. S. Ahn et al. , 2010, Measurements of the relative abundances of high-energy cosmic-ray nuclei in the TeV/Nucleon region, ApJ, 715, 1400-1407.

[13] J. A. Simpson (Experiment HEAO-C2), Elemental and Isotopic Composition of the Galactic Cosmic Rays, 1983, Ann. Rev. of Nucl. and Part. Science, 33, 323-382.

[14] B. Wiebel-Sooth et al., 2009, Cosmic Rays VII. Individual element spectra: prediction and data, [astro-ph/0909.0948v1].

[15] M. Asplumb et al., 2009, The chemical composition of the Sun [arXiv: $0909.0948 v 1$ ]

[16] Katharina Lodders, 2003, Solar System Abundances and Condensation Temperatures of the Elements, ApJ, 591, 1220-1247.

[17] Priscilla C. Frisch et al. ,2011, The Interstellar Medium Surrounding the Sun, Annual Review of Astronomy and Astrophysics, 2011, Vol. 49, pages 237-279.

[18] T. P. Snow and A. N. Witt, 1996, Interstellar depletion updated : where all the atoms went, ApJ Letters, L65-L68.

[19] B. Edvardsson et al., 1993, The chemical evolution of the galactic disk, I. Analysis and results, Astronomy and Astrophysics, 275, 101-152.

[20] A. Codino, 2015, The dominance of secondary nuclei in the cosmic radiation and the modulation of nuclear species at the injection of the galactic accelerator, in proceedings of 34th ICRC, work 038, The Hague, Netherlands.

[21] D. A. Verner et al., 1996, Atomic Data for Astrophysics. II. Analytical Fits for Photoionization Cross Sections of Atoms and Ions, [arXiv: astro-ph/ 96011009v2] .

[22] R. J. W. Henry, 1970, The Photoionization cross-section for atoms and ions of Carbon, Nitrogen, Oxygen, and Neon, ApJ, 161, 1153-1155.

[23] J. B. Kaler e W. A. Feibelman, 1985, The remarkable ultraviolet spectra of central stars of large planetary nebula, ApJ, 277, 716-724.

[24] K. Kristiansson, 1972, Further Evidence for a Cosmic Ray Selection Mechanism, Astrophys. Space Science 16, 405-412.

[25] O. Havnes, 1971, On cosmic rays and Magnetic stars, Nature, 229, 548.

[26] M. E. Wiedenbeck et al., 1999, Constrains on the time delay between nucleosynthesis and cosmic-ray acceleration from observation of 59Ni and 59Co, ApJ Letters, 523, pages L61-L64. 